\title{
Multi-Scale Simulation of Trickle Bed Reactor’s Liquid-Solid Mass Transfer
}

\author{
Yixuan Yin ${ }^{\mathrm{a}}$, Zuoguo Yang ${ }^{\mathrm{b}, *}$ \\ School of Chemical Engineering, East China University of Science and Technology, 130 Meilong Road, \\ Xuhui District, Shanghai, 200237, P.R. China. \\ a13585617721@163.com, bgyang@ecust.edu.cn
}

Keywords: Trickle-bed reactor; multiphase flow; mass transfer; CFD simulation

\begin{abstract}
Trickle bed reactor (TBR)'s liquid-solid mass transfer and liquid outflow's tracer concentration curves were simulated by coupling internal mass transfer data, external diffusion model and macro TBR computational fluid dynamic (CFD) model. The predicted Peclet $(\mathrm{Pe})$ numbers and liquid outflow's tracer concentration curves were fitting well with the experiment results. Simulation results show that the mean relative deviation of the predicated $P e$ value is less than $8 \%$. Higher liquid flow rate leads to a steeper concentration curve and early breakthrough. While the effects of gas flow rate on outflow tracer concentration curves are not obvious. Tracer concentration contours indicate that there was a uniform flow distribution in TBR when the liquid flow rate was high.
\end{abstract}

\section{Introduction}

Trickle bed reactor (TBR) is a kind of multi-phase catalytic reaction device where liquid and gas flow through a porous fixed bed concurrently or counter-currently. TBRs were widely applied in petrochemical processes such as hydrodesulphurization [1], hydrogenation synthesis [2] and catalytic oxidation [3]. Although the application of TBRs has a long history, TBR's design and scaling up are still challenging, and more efforts should be made to improve TBR simulation technique.

Under different gas or liquid superficial velocities, particle shapes and fluid properties, TBRs can be operated hydro-dynamical differently [4]. The present paper intends to simulate high liquid flux trickle bed reactor's multi-scale mass transfer behaviors by means of a micro-macro mass transfer coupled Eulerian simulation approach. The basic simulation process was as follows. First, the microcosmic interior tracer mass transfer data base was established by an auxiliary simulation. Then, a searching and interpolation algorithm were developed to create a correspondence between tracer concentration and tracer accumulation rate in TBR's static phase, and the crude result was rectified based on external mass transfer theories. Above data and algorithm were loaded in macro multiphase flow CFD case. Finally, an overall simulation was carried out and mass transfer data was generated.

\section{Mass transfer modeling}

In this study, a liquid flow contains tracer will continuously enter into a TBR device after its flow field has fully developed. The tracer distribution function's curves were acquired by measuring mass flow rate weighted average tracer concentration at reactor's outlet flow. The simulated trickle bed device was $0.152 \mathrm{~m}$ in diameter and $1.25 \mathrm{~m}$ in height. Potassium chloride served as the tracer and injected into the upstream of the column. It's equivalent mass transfer coefficient in porous alumina particles was $D_{\mathrm{t}, \mathrm{s}}=0.563 \times 10^{-9}\left[\mathrm{~m} 2 \cdot \mathrm{s}^{-1}\right]$. Detailed parameters can be acquired in Nigam's study [5].

\subsection{Interior mass transfer}

In order to save calculation time, the sphere particles were simplified into a cone like model, which take static liquid into consideration. Mass conservation equation was included to simulate tracer's diffusion in alumina particles. Where $\varepsilon_{1}$ is particle's porosity.

$$
\frac{\partial_{\mathrm{t}, \mathrm{s}}}{\boldsymbol{\partial}}+\nabla \cdot\left(D_{\mathrm{t}} \varepsilon_{1} \nabla c_{\mathrm{t}, \mathrm{s}}\right)=0
$$


Resulting tracer concentration distribution in particle pores for specific time was showed in Fig 1.

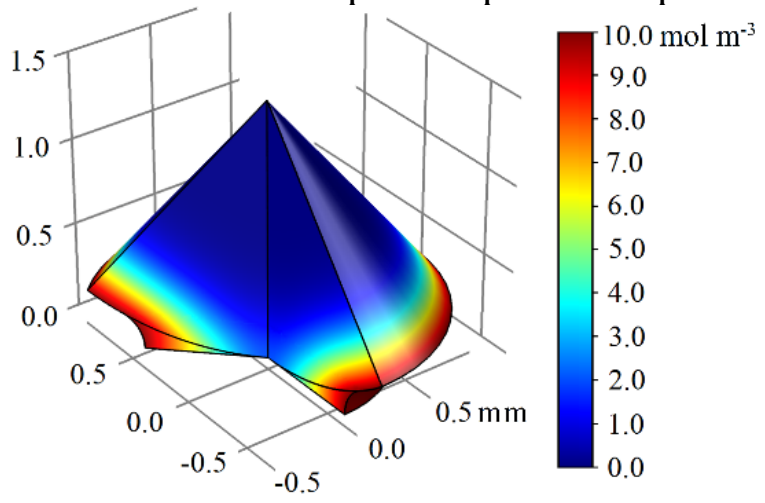

Fig 1. Tracer concentration distribution in particle $(\mathrm{t}=60 \mathrm{~s})$

On the basis of the internal mass transfer simulation, a dispersing relationship between averaged tracer concentration in particle, mass transfer rate for per and their derivative respect to time were acquired. In unit volume of reactor, alumina particle’s tracer absorption rate can be expressed as:

$$
A_{\text {trans }} D_{\text {t,eff }}\left(\frac{\partial C_{t, s}}{\partial t}\right)_{\text {surface }}=\left(1-\varepsilon_{1}\right) \frac{d C_{t, s, a v e}}{d t}
$$

Searching algorithm was adopted to find the tracer concentration interpolation points whose value were merely smaller or larger than given $c_{\mathrm{t}, \mathrm{s}, \mathrm{ave}}$. The alumina particles' tracer absorption rate for any given averaged tracer concentration in particle $c_{\mathrm{t}, \mathrm{s}, \text { ave }}$ were acquired by Hermite interpolation.

\subsection{External Mass Transfer}

The external mass transfer resistance could be attributed to the limited tracer flux through particle's surface. The value of external mass transfer coefficient $k_{\mathrm{m}}$ can be calculated by the correlations given by Dwivedi and Upadhyay [6].

$$
\varepsilon_{1} j_{\mathrm{D}}=\frac{0.765}{\left(\operatorname{Re}_{1}\right)^{0.82}}+\frac{0.365}{\left(\operatorname{Re}_{1}\right)^{0.386}}=\frac{k_{\mathrm{m}} a\left(u_{\mathrm{g}} \rho_{\mathrm{g}}+u_{1} \rho_{1}\right)}{(L+G)}\left[\frac{u_{\mathrm{g}} \mu_{\mathrm{g}}+u_{1} \mu_{1}}{\left(u_{\mathrm{g}} \rho_{\mathrm{g}}+u_{1} \rho_{1}\right) D}\right]^{2 / 3}
$$

External mass transfer's overall effect can be descripted by the external efficient factor $\eta_{\mathrm{e}}$, whose value could be deducted from chemical reaction order and Da (Damköhler) number. In this study, particles' tracer absorption process was treated as a hypothetic tracer consume chemical reaction with modifiable reaction order and fixed pre-exponential factor.

$$
\begin{gathered}
D a=\frac{k c_{\mathrm{t}, \mathrm{s} \text { ave }}^{\mathrm{n}}}{k_{\mathrm{m}} a\left(c_{\mathrm{t}, \mathrm{l}}-0\right)} \\
k c_{\mathrm{t}, \mathrm{s}, \mathrm{ave}}^{\mathrm{n}}=r_{\mathrm{t}}^{\prime}=\frac{\partial c_{\mathrm{t}, \mathrm{s}, \mathrm{ave}}}{\partial t}
\end{gathered}
$$

Where, $r_{\mathrm{t}}$ was the hypothetic chemical reaction rate which describing the tracer consumption in dynamic liquid, $k$ was reaction coefficient, $n$ was the reaction order. The hypothetic reaction coefficient and reaction order can be deduced as follows.

$$
k c_{\mathrm{t}, \mathrm{s}, \mathrm{av}, 0}^{\mathrm{n}}=r_{\mathrm{t}, 0}^{\mathrm{j}}=\left.\frac{\boldsymbol{\partial}_{\mathrm{t}, \mathrm{s}, \mathrm{ave}}}{\boldsymbol{\partial}}\right|_{t=0}
$$

Where $r_{\mathrm{t}, 0}$ means the initial tracer absorption rate. When the virgin particles contact the inlet flow, the maximum tracer absorption rate can be measured and the $r_{\mathrm{t}, 0}$ acquired. External mass transfer efficient factor and liquid-solid mass transfer rate was given by the following expressions:

$$
\eta_{\mathrm{e}}=\left(1-\eta_{\mathrm{e}} D a\right)^{\mathrm{n}}
$$




\subsection{CFD Modeling Framework}

In this work, Euler-Euler framework was adopted to present CFD equations. The continuity equation, mass balance equation and momentum balance for each phase were written as follows [7]:

$$
\begin{gathered}
\frac{\partial_{\mathrm{q}} \rho_{\mathrm{q}}}{\partial}+\nabla \cdot\left(\varepsilon_{\mathrm{q}} \rho_{\mathrm{q}} u_{\mathrm{q}}\right)=\sum_{\mathrm{p}=1}^{\mathrm{n}}\left(\mathrm{m}_{\mathrm{pq}}\right)+S_{\mathrm{i}, \mathrm{q}} \\
\frac{\partial_{\mathrm{q}} \rho_{\mathrm{q}} C_{\mathrm{t}, \mathrm{q}}}{\partial}+\nabla \cdot\left(\varepsilon_{\mathrm{q}} \rho_{\mathrm{q}} \boldsymbol{u}_{\mathrm{q}} c_{\mathrm{t}, \mathrm{q}}\right)=-\nabla \cdot\left(\varepsilon_{\mathrm{q}} D_{\mathrm{t}, 1} \nabla c_{\mathrm{t}, \mathrm{q}}\right)+\sum_{\mathrm{p}=1}^{\mathrm{n}}\left(\mathrm{m}_{\mathrm{pq}} c_{\mathrm{t}, \mathrm{q}}\right)+a_{\mathrm{q}} S_{\mathrm{t}, \mathrm{q}} \\
\frac{\partial_{\mathrm{q}} \rho_{\mathrm{q}} \boldsymbol{u}_{\mathrm{t}, \mathrm{q}}}{\partial}+\nabla \cdot\left(\varepsilon_{\mathrm{q}} \rho_{\mathrm{q}} \boldsymbol{u}_{\mathrm{q}} \boldsymbol{u}_{\mathrm{q}}\right)=-\varepsilon_{\mathrm{q}} \nabla p+\nabla \cdot \bar{\tau}_{\mathrm{q}}+\mathrm{a}_{\mathrm{q}} \rho_{\mathrm{q}} \boldsymbol{g}+\sum_{\mathrm{p}=1}^{\mathrm{n}}\left(f_{\mathrm{pq}}+\mathrm{m}_{\mathrm{pq}} \boldsymbol{u}_{\mathrm{pq}}\right)
\end{gathered}
$$

$c_{\mathrm{t}, \mathrm{q}}$ and $s_{\mathrm{t}, \mathrm{q}}$ represent for the concentration and source term of tracer in $q$ phase respectively, $\mathrm{m}$ was the interphase mass exchange term, $\varepsilon_{\mathrm{q}}$ means q phase's volume fraction, $\rho_{\mathrm{q}}$ was q phase's density, $\boldsymbol{u}_{\mathrm{q}}$ was q phase's velocity vector. $D_{\mathrm{t}, 1}$ was tracer's effective dispersion coefficient.

$\mathrm{m}_{\mathrm{pq}}$ was the mass transfer rate and $f_{\mathrm{pq}}$ was the drag force between phase $\mathrm{p}$ and $\mathrm{q}$. Attou's bubble flow model [8] was applied in this CFD simulation to close momentum balance equations. Chung and Wen's correlation [7] was used to model the hydromechanics dispersion. A correlation given by Klerk [9] were hooked in the CFD computation so as to simulate TBR's radial porosity distribution.

\subsection{CFD computation}

Multiphase flow in trickle bed was simulated in axis-symmetric frame. It was verified that 400 grids in axial direction and $\mathbf{4 5}$ grids in radial direction on the domain are sufficient to achieve grid independent results. Unsteady simulations were carried out with the time steps ranging from $0.001 \mathrm{~s}$ to 0.01s. Semi-Implicit Method for Pressure Linked Equations (SIMPLE) was implemented for evaluating pressure and velocity coupling. Least squares cell based spatial discretization were used to disperse gradient. while quadratic upwind interpolation (QUICK) method were used to solve momentum, phase volume fraction and tracer mass transport equation.

\section{Results and discussions}

The numerical predictions resulted from the present model were validated against available mass transfer experiment data of a co-current downward flow trickle bed reactor. In table 1, the predicted $\mathrm{Pe}$ (Peclet) number were compared with the experimental results [5].

Table 1 Predicated and experimental Pe (Peclet) numbers

\begin{tabular}{cccc}
\hline $\begin{array}{c}j_{\mathrm{g}} \\
{\left[\mathrm{mm} \cdot \mathrm{s}^{-1}\right]}\end{array}$ & $\begin{array}{c}j_{\mathrm{l}} \\
{\left[\mathrm{mm} \cdot \mathrm{s}^{-1}\right]}\end{array}$ & $\begin{array}{c}P e \\
\text { Predicted results }\end{array}$ & $\begin{array}{c}P e \\
\text { Experiment results }\end{array}$ \\
\hline 0 & 9.4 & 31.63 & 33 \\
9.1 & 9.4 & 38.32 & 37 \\
27.4 & 9.4 & 39.67 & 37 \\
0 & 13.7 & 50.98 & 50 \\
9.1 & 13.7 & 51.14 & 49 \\
27.4 & 13.7 & 54.19 & 51 \\
0 & 18.3 & 58.68 & 50 \\
9.1 & 18.3 & 48.19 & 50 \\
18.3 & 18.3 & 51.41 & 58 \\
27.4 & 18.3 & 48.23 & 58 \\
\hline
\end{tabular}

As can be seem in above table, the flow rate of liquid and gas flow had a direct effect on Pelect number. The higher gas/liquid inlet superficial velocity leaded to higher Pelect number. Gas phase flow velocity's effect was no as significant as liquid phase's. The predicted Pe number's averaged relative deviation is $7.7 \%$. This simulation results are generally consistent with the experiment data.

When the tracer concentration in TBR's inlet liquid keeps constant, the outflow's tracer concentration could be simulated by the mass transfer model introduced above. The trarcer 
concentration curves for various operation conditions were displayed in Fig. 1 and Fig. 2. As a comparison task, Nigam's model was also solved in this study. This model is a parabolic partial differential equation set, whose parameters were fitted by experimental data [5].

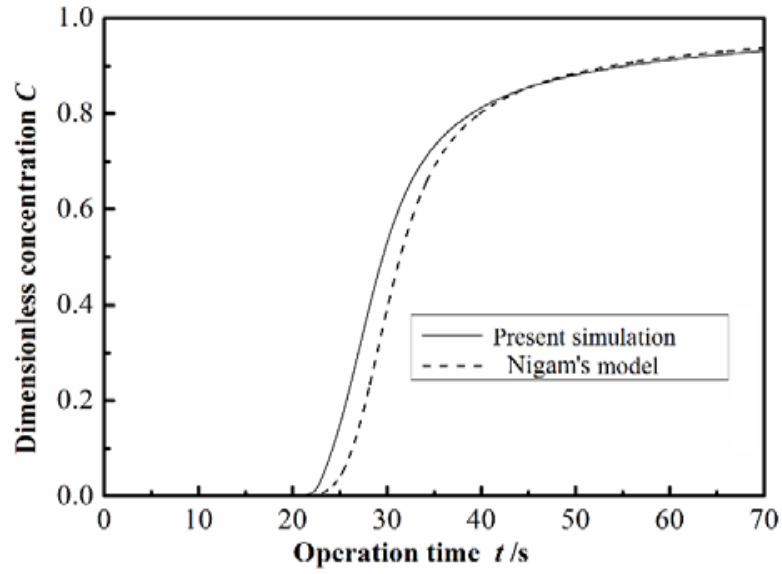

(a)

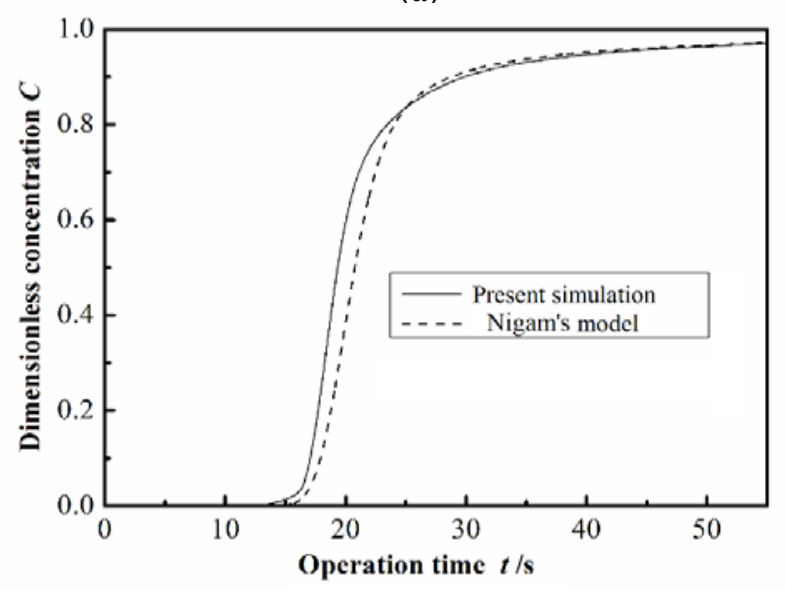

(c)

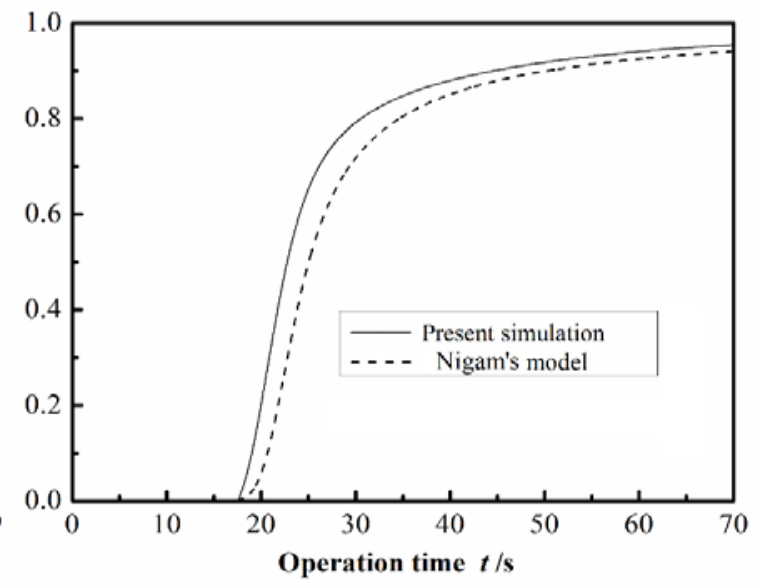

(b)

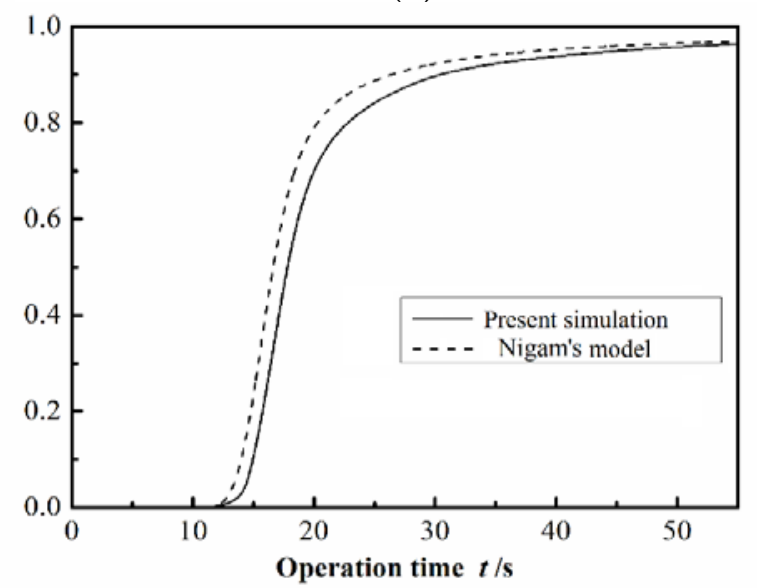

(d)

Fig. 2 The outlet tracer concentration curves of TBR
(a), $j_{1}=0.0094 \mathrm{~m} \cdot \mathrm{s}^{-1} j_{\mathrm{g}}=0.0091 \mathrm{~m} \cdot \mathrm{s}^{-1}$
(b), $j_{1}=0.0094 \mathrm{~m} \cdot \mathrm{s}^{-1} j_{\mathrm{g}}=0.0274 \mathrm{~m} \cdot \mathrm{s}^{-1}$
(c), $i_{1}=0.0183 \mathrm{~m} \cdot \mathrm{s}^{-1} \quad i_{\mathrm{o}}=0.0091 \mathrm{~m} \cdot \mathrm{s}^{-1}$
(d), $i_{1}=0.0183 \mathrm{~m} \cdot \mathrm{s}^{-1} i_{\mathrm{o}}=0.0274 \mathrm{~m} \cdot \mathrm{s}^{-1}$

The liquid outflow's tracer concentration curves generated in this study are fitting well with the parabolic partial differential model whose coefficients were fitted by experiments ${ }^{[5]}$. Higher liquid flow rate leads to a steep concentration curve and early breakthrough. While the effects of gas flow rate on concentration curves were not as obvious as liquid flow rates'. The location of curves' zoomac were also close to Nigam's model, which indicates that the predicated liquid holdup also agrees well with experiment results.

The simulation procedure generated TBR device' overall mass transfer data. When $j_{1}=0.0094$ $\mathrm{m} \cdot \mathrm{s}^{-1} j_{\mathrm{g}}=0.0091 \mathrm{~m} \cdot \mathrm{s}^{-}$, the typical dynamic and solid phase's mass concentration contours of TBRs filled with sphere alumina particles $(D=3 \mathrm{~mm})$ were displayed in Fig. 3and Fig. 4.

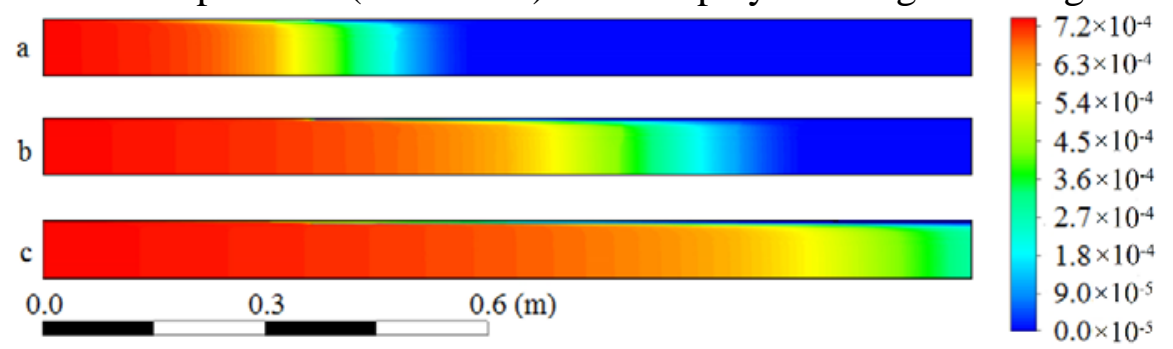

Fig. 3 Tracer mass fraction contours in liquid phase a), t $=7.5 \mathrm{~s} \mathrm{~b}), \mathrm{t}=15.0 \mathrm{~s} \mathrm{c}$ ), $\mathrm{t}=22.5 \mathrm{~s}$. 


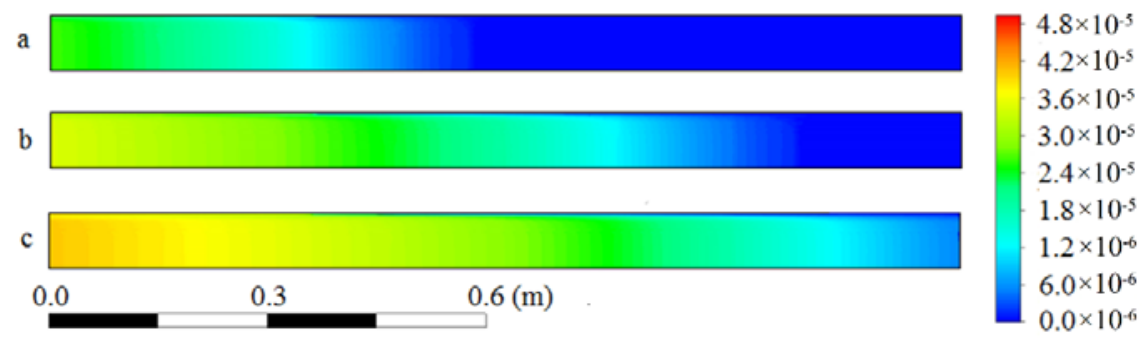

Fig. 4 Tracer mass fraction contours in particles a), $\mathrm{t}=7.5 \mathrm{~s} \mathrm{~b}), \mathrm{t}=15.0 \mathrm{~s} \mathrm{c}$ ), $\mathrm{t}=22.5 \mathrm{~s}$.

As can be seen in Fig. 3 and 4, the tracer has a uniform concentration distribution in bed's radial direction. The effect of radial voidage distribution in bed is not obvious in this study, which indicates that the high liquid flux operation could improve the multiphase flow distribution in TBRs.

\section{Conclusions}

CFD simulations of a high liquid flux TBR's mass transfer were carried out by means of Eulerian-Eulerian multiphase model. The micro-macro combined mass transfer simulation approach was established and tested.

The predicted $P e$ numbers were fitting well with the experiment results. The average relative deviation is $7.7 \%$. Higher gas/liquid inlet superficial velocity leaded to higher $P e$ number. Gas phase's inlet velocity's effect on $P e$ number was no as significant as liquid phase's. Higher liquid flow rate lead to a steeper tracer concentration curve and early breakthrough. While the effects of gas flow rate on concentration curves are not obvious. The predicated liquid outflow tracer concentration curves agree well with the experiment fitted axial dispersion model. The results show that the effects of radial voidage distribution in TBR bed could be neglected under high liquid flux operation.

\section{References}

[1] V. Andreas, P. Nikos, Hydrodesulphurization and hydrodeoxygenation of gasoil-vegetable oil mixtures over a Pt/ $\gamma-\mathrm{Al}_{2} \mathrm{O}_{3}$ catalyst, J. Fuel Processing Technology. 150 (2016) 126-131.

[2] K. Teuvo, B. Pierdomenico, B. Alice, et, al. Modeling of direct synthesis of hydrogen peroxide in a packed-bed, J. Ind. Eng. Chem. Res. 51 (2012) 13366-13378.

[3] A. Singh, K.K. Pant, K.D.P. Nigam.Catalytic wet oxidation of phenol in a trickle bed reactor, J. Chemical Engineering Journal. 103 (2004) 51-57.

[4] R.J.G. Lopes, M Rosa. Quinta-Ferreira Trickle-bed CFD studies in the catalytic wet oxidation of phenolic acids, J. Chemical Engineering Science. 62 (2007) 7045-7052.

[5] K.D.P. Nigam, I. Iliuta, F. Larachi, Liquid back-mixing and mass transfer effects in trickle-bed reactors filled with porous catalyst particles, J. Chemical Engineering and Processing. 41 (2002) 365-371.

[6] P.N. Dwivedi, S.N. Upadhyay, Particle-fluid mass transfer in fixed and fluidized beds, J. Ind. Eng. Chem. Process Des. Dev. 16 (1977) 157-165.

[7] S.F. Chung, C.Y. Wen, Longitudinal dispersion of liquid flowing through fixed and fluidized beds, J. AIChE J. 14 (1968) 857-866.

[8] A. Attou, G. Ferschneider. A simple model for pressure drop and liquid hold-up in packed-bed bubble reactors, J. Chemical Engineering Science. 54 (1999) 5139-5144.

[9] A. Klerk, Voidage variation in packed beds at small column to particle diameter ratio, J. AIChE J. 49(8) (2003) 2022-2029. 